\title{
Free-Space Optical Interconnects for Cable-less Readout in Particle Physics Detectors
}

\author{
John Chramowicz ${ }^{a}$, Simon Kwan ${ }^{a}$, Tony Moretti ${ }^{b}$, Alan Sugg ${ }^{b}$, and Alan Prosser ${ }^{a, 1}$ \\ ${ }^{a}$ Fermi National Accelerator Laboratory, Pine Street, Batavia, IL 60510, USA \\ ${ }^{b}$ Vega Wave Systems, 1275 W. Roosevelt Rd, Suite 104, West Chicago, IL 60185, USA \\ E-mail: aprosserefnal.gov
}

\begin{abstract}
Particle physics detectors utilize readout data links requiring a complicated network of copper wires or optical fibers. These links are both massive and costly. Upgrades to such detectors may require additional bandwidth to be provisioned with limited space available to route new cables or fibers. In contrast, free-space optical interconnects will offer cable-less readout, thereby resulting in significant reductions of material and labor. A collaborative effort between Fermilab and Vega Wave Systems is pursuing the development of a unique free-space optical link design that utilizes the transparency of silicon at wavelengths including $1310 \mathrm{~nm}$ and multiple wavelengths used in standard telecommunications applications such as coarse wavelength division multiplexing (CWDM). The first step in the pursuit of that design is a proof that the concept may be viable. To that end, experiments have been performed to characterize the bit error rate performance of a prototype link over a free-space optical path and through doped silicon at multi-gigabit rates. These experiments have demonstrated that operation within acceptable bit error rates is possible using single and multiple wavelength transmission arrangements.
\end{abstract}

KEYWORDS: Optical data transmission, wavelength division multiplexing, detector readout systems.

This work was supported by the U.S. Department of Energy, operated by Fermi Research Alliance, LLC under contract No. DE-AC02-07CH11359 with the United States Department of Energy.

\footnotetext{
${ }^{1}$ Corresponding Author
} 


\section{Contents}

1. Introduction $\quad 1$

2. Experiment Descriptions: $\quad 2$

2.1 CWDM Experiment $\quad 2$

$\begin{array}{ll}\text { 2.2 Single Wavelength Experiment } & 3\end{array}$

3. Results $\quad 3$

4. Summary $\quad 5$

\section{Introduction}

Modern particle physics experiments are characterized by massive data readout requirements. A state of the art experiment is typically made up of a detector with multiple sub-detectors, all operating to deliver physics data resulting from the collisions of particles produced in an accelerator. The transmission of this data requires a substantial infrastructure of electrical cables and optical fibres. These components represent a significant fraction of the mass inside the detector volume. However, the science goals of high energy physics experiments dictate that the material budget inside the detector volume be reduced as much as possible. This reduces the multiple scattering and nuclear interactions from the material in the communications subsystem. It also reduces the burden of management of the cable or wire masses.

Fermilab and Vega Wave Systems have embarked upon an investigation of the use of cable-less techniques for the operation of data links inside the detector. These techniques seek to take advantage of free-space transmission using standard transceiver components operating in excess of 1 Gigabit per second (Gbps) rates. The use of wavelengths that are commonly employed in telecommunications systems makes possible the selection of commercially available components for a feasibility study including a proof of the concept in the laboratory.

In addition to the use of free-space techniques, a goal of this experiment was to examine the possibility of using through-silicon transmission as well. If the transmission of an optical signal can be accomplished through the silicon in a detector, it may reduce the need to identify line of sight paths free of any material at all within the detector volume. Figure 1 below illustrates the result of a test carried out at Vega Wave Systems that demonstrates the transmission of infrared wavelengths through a sample of doped silicon. The flat characteristic of the transmission spectrum shows that the material is equally transparent for wavelengths within the range illustrated. The wavelength range of the experiment includes wavelengths between $1470 \mathrm{~nm}$ and $1530 \mathrm{~nm}$. This is significant because optical transceivers which operate within this range are readily available and can be selected compatibly with CWDM multiplexers and de-multiplexers. 


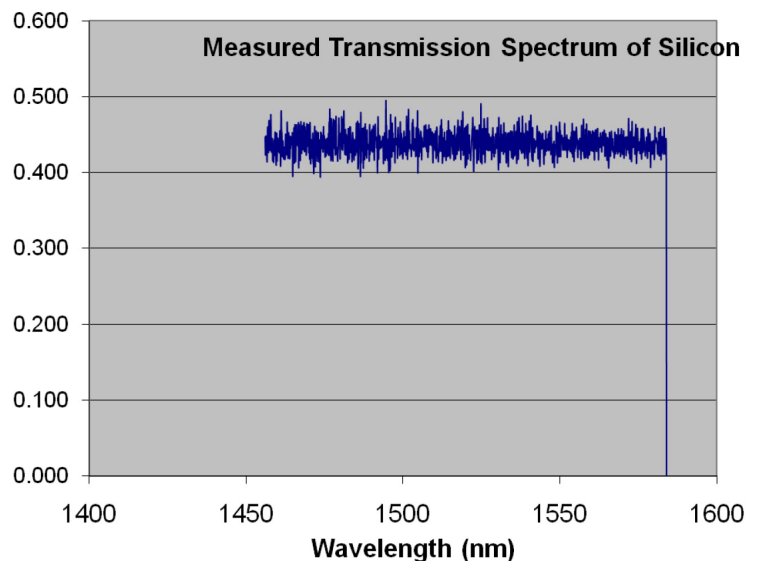

Figure 1: Transmission of light through approximately $2 \mathrm{~mm}$ of doped silicon over a free-space optical path of approximately $60 \mathrm{~cm}$. The y-axis is the fraction of launched power measured at the receiving end of the link

\section{Experiment Descriptions}

\subsection{CWDM Experiment}

Figure 2 below illustrates one of the experimental configurations used to implement a test of the concept. Four SFP optical transceivers were used to serve as transmitters and receivers of a pseudorandom bit stream (PRBS). The transceivers were selected to be compatible with the use of CWDM multiplexers and de-multiplexers from JDSU, Milpitas,CA, USA. By employing these components, it was possible to combine four wavelengths at $1470 \mathrm{~nm}, 1490 \mathrm{~nm}, 1510 \mathrm{~nm}$, and $1530 \mathrm{~nm}$ respectively. Each transceiver was operated at $1 \mathrm{Gbps}$ resulting in an aggregate bit rate of 4 Gbps.

The output of the common fibre of the CWDM multiplexer is delivered to a lens for launching the light over a free-space optical path. Within the optical path, two sections of doped silicon may be introduced. Each section is approximately $2 \mathrm{~mm}$ in thickness.

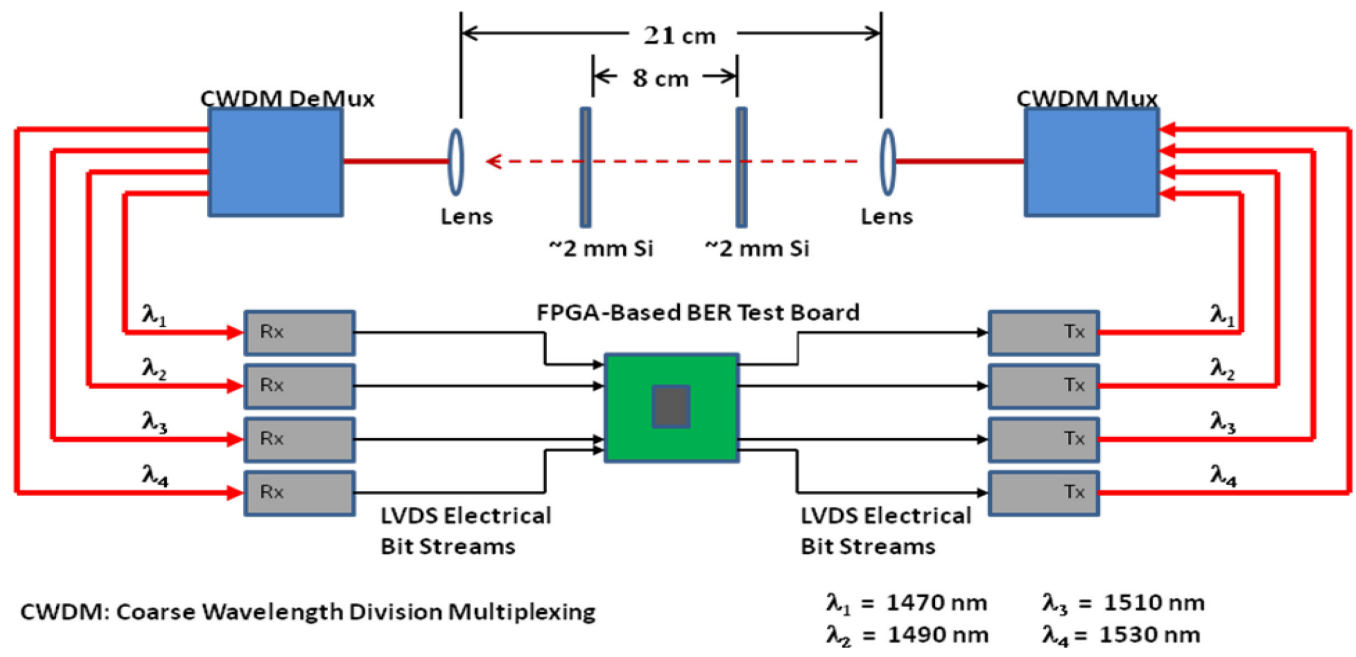


Figure 2: Block diagram of the experimental setup for a test of coarse wavelength division multiplexing over a free-space link.

The PRBS data was sourced using an FPGA-based (Stratix II) signal integrity kit from Altera. The use of this approach makes possible bit error rate tests (BERT) at a reasonable cost. All four channels indicated were driven at 1 Gbps using a PRBS stream $\left(2^{7}\right)$ without any special encoding. The FPGA on the signal integrity board generates the PRBS stream and collects the received data after the link so that it can be checked for bit errors. Error counters on the graphical user interface for the signal integrity kit report individual errors and can be used to generate an estimate of the link bit error rate.

Figure 3 is a photograph of the hardware used to implement the free-space link.

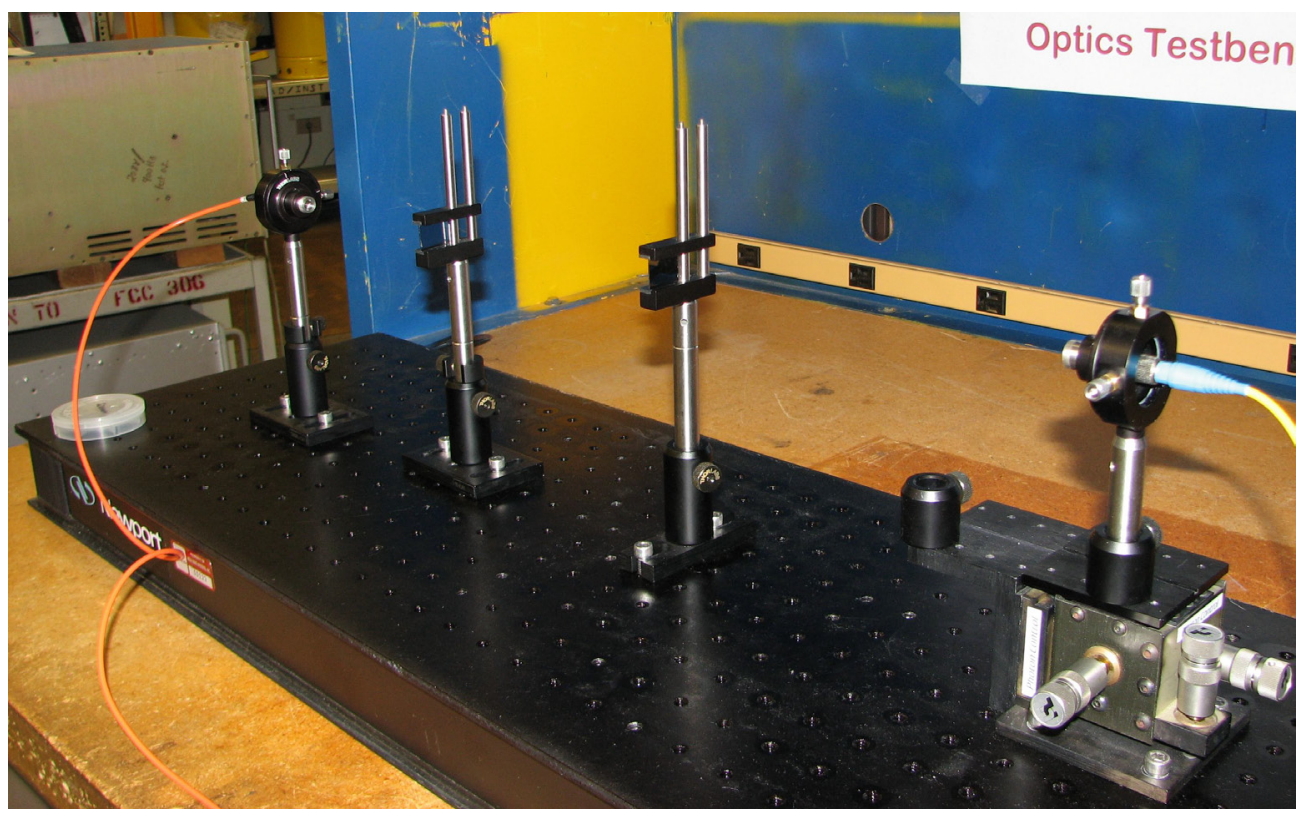

Figure 3: Free-space optical path implemented on an optical breadboard. On the right is the transmitter end with single mode fibre and launching lens. On the left is the receiver end with multimode fibre and receiving lens. In between are two swinging gates with doped silicon samples.

\subsection{Single Wavelength Experiment}

A similar set up, illustrated in Figure 4, was used to test the transmission of data at a single wavelength $(1310 \mathrm{~nm})$ but at higher bit rates. For this test, no CWDM components were needed. In addition, to model the distances that might span a communications link in a particle physics experiment (from the detector to front-end electronics racks off-detector), a spool of 100 meters of multimode fibre was placed after the free-space optical path. The use of multimode fibre at the receiving end is intended to improve the reception of optical power as the core diameter of the multimode fibre is $50 \mu \mathrm{m}$ (as opposed to $9 \mu \mathrm{m}$ for single mode fibre). Tests were carried out at bit rates including $3.125 \mathrm{Gbps}, 6.25 \mathrm{Gbps}$, and $10 \mathrm{Gbps}$. The testing at $10 \mathrm{Gbps}$ was made possible with a different signal integrity board based upon the Altera Stratix IV GT FPGA. 


\section{Results}

Both the CWDM and single wavelength experiments were operated without errors for resulting in bit error rates in excess of $10^{-12}$, a common standard of acceptable links. However, the intro-

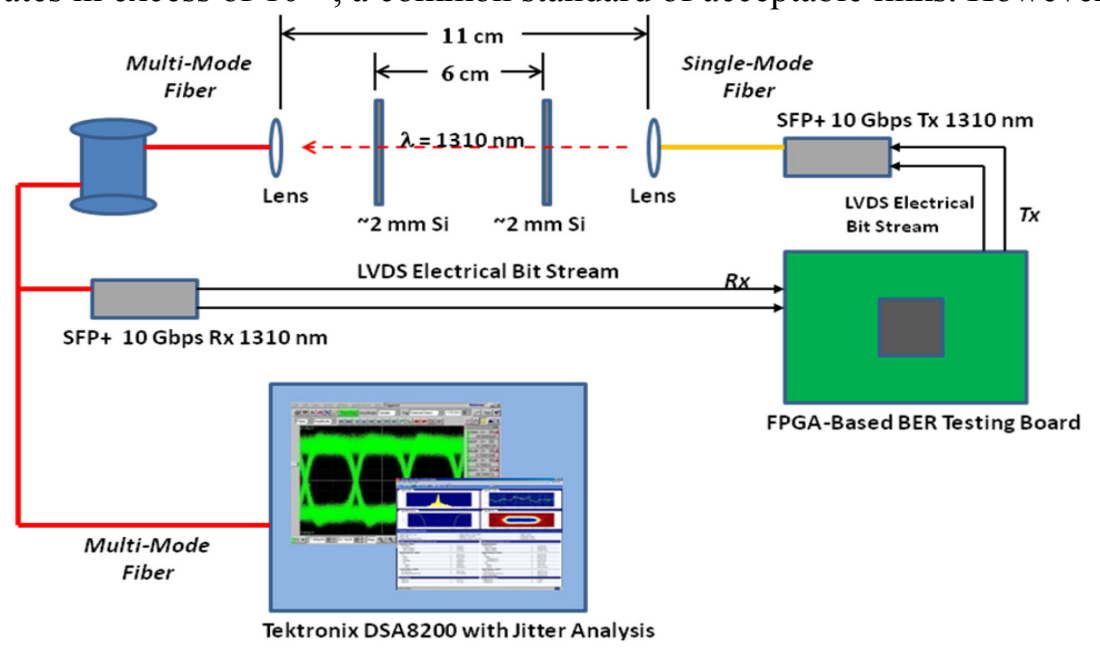

Figure 4: Block diagram of the experimental setup for a test of coarse wavelength division multiplexing over a free-space link.

duction of a third slice of silicon resulted in the onset of multiple bit errors. This behavior is probably due to the additional reflection losses that result from the additional air-silicon interface that is added when another silicon slice is added. The silicon slices were not treated with anti-reflective coating so some reflection on the order of $47 \%$ per slice would be expected.

Figure 5 illustrates the effect of transmission over free-space link with and without silicon. The eye diagrams show the degradation in received average power (AOP).
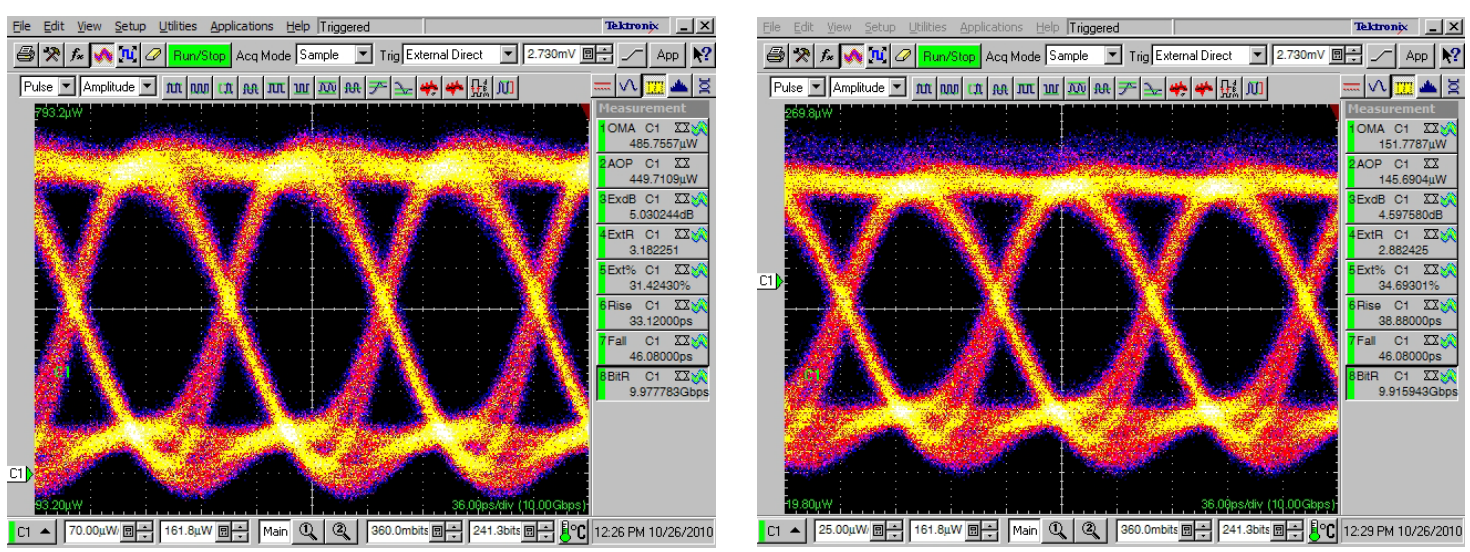

Figure 5: Eye diagrams of optical signal without (left) and with (right) two silicon slices in the free-space link. These signals were generated at $10 \mathrm{Gbps}$ and supported bit error rates better than $10^{-12}$.

The additional silicon reduces the AOP from $450 \mu \mathrm{W}$ to $150 \mu \mathrm{W}$. Even in the presence of this signal degradation, the system was able to operate with no bit errors for hours at a time. 


\section{Summary}

The tests described in this paper indicate that further effort to develop free-space optical links is worth investing. Work is presently underway to further explore the possibilities of this approach and to determine the limits required of the optical and mechanical design of a system based on these techniques. The experiments described in this paper are but a first step and will require a significant effort to carry through to a feasible design for use in a future particle detector. Figure 6 illustrates a conceptual design that might include transmission to off-detector receivers using multiple wavelengths to identify different detector layers without the need for address bits.

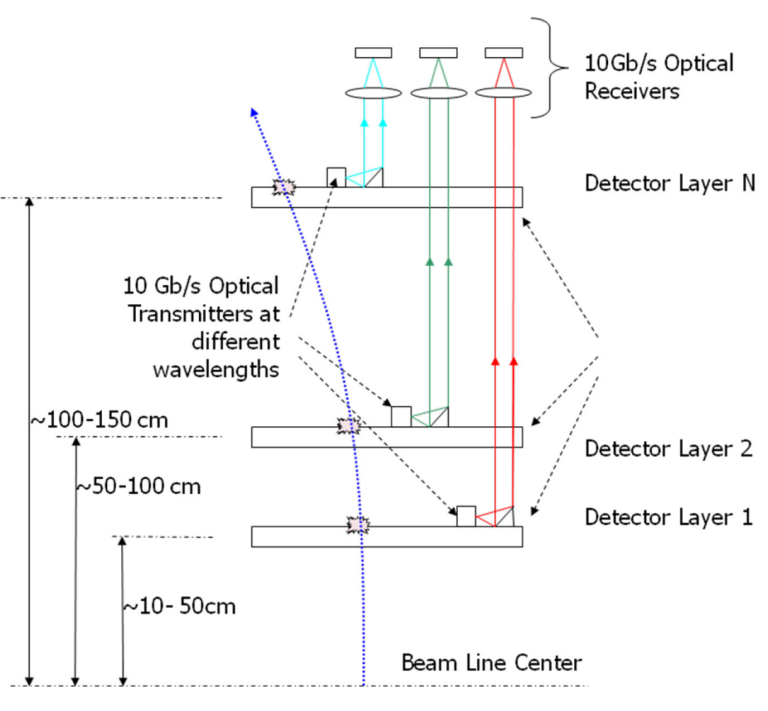

Figure 6: Conceptual design of future free-space optical transmission system for particle physics detector. The path of a particle is indicated as a dotted blue line. This excites signals in the layers of the detector and the resulting data is transmitted using multiple wavelengths (e.g. red for the innermost layer, green for the next layer) and reflected by micro-mirrors to optical receivers outside the detector volume.

The next step is to refine the design of an optical system including controls for alignment that would be suitable for use in the actual detector. This requires consideration of the mechanical and thermal stability requirements as well as the free space optical path lengths and any constraints imposed by the limited space available within the detector volume.

\section{Acknowledgments}

The authors would like to acknowledge Orlando Colon and Chuck Andrews of Fermilab who assisted in the procurement of components used in these tests. Annie Xiang of Southern Methodist University provided SFP/SFP+ test boards used to host the optical transceivers.

\section{References}

[1] J. Troska et al., Optical Readout and Control Systems for the CMS Tracker, IEEE Transactions on Nuclear Science, Vol. 50, No. 4, August 2003. 